\title{
Swirl sign in epidural hematoma
}

\section{Sinal do redemoinho no hematoma epidural}

Marcos Rosa Júnior?', Larissa Aguiar Martins², Laiza Alcure Dias Scussulin ${ }^{3}$

A 32-year-old male was admitted to the emergency room due to traumatic brain injury. The computed tomography (CT), three hours after the trauma, showed an epidural hematoma with the swirl signal. After 24 hours the patient evolved with worsening of the condition and the CT was repeated (Figure).
The swirl signal is described as an area of low attenuation within an hyperattenuating hematoma ${ }^{1}$. Recent studies indicate that the swirl signal is a direct sign of hematoma expansion, associated with high morbidity and mortality rates ${ }^{1}$. Unlike the spot sign, another predictor of epidural hematoma expansion ${ }^{2-5}$, the swirl signal does not require the use of contrast.

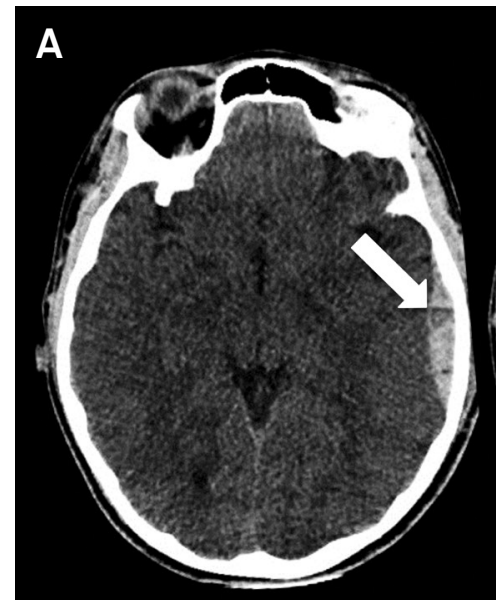

B C

D
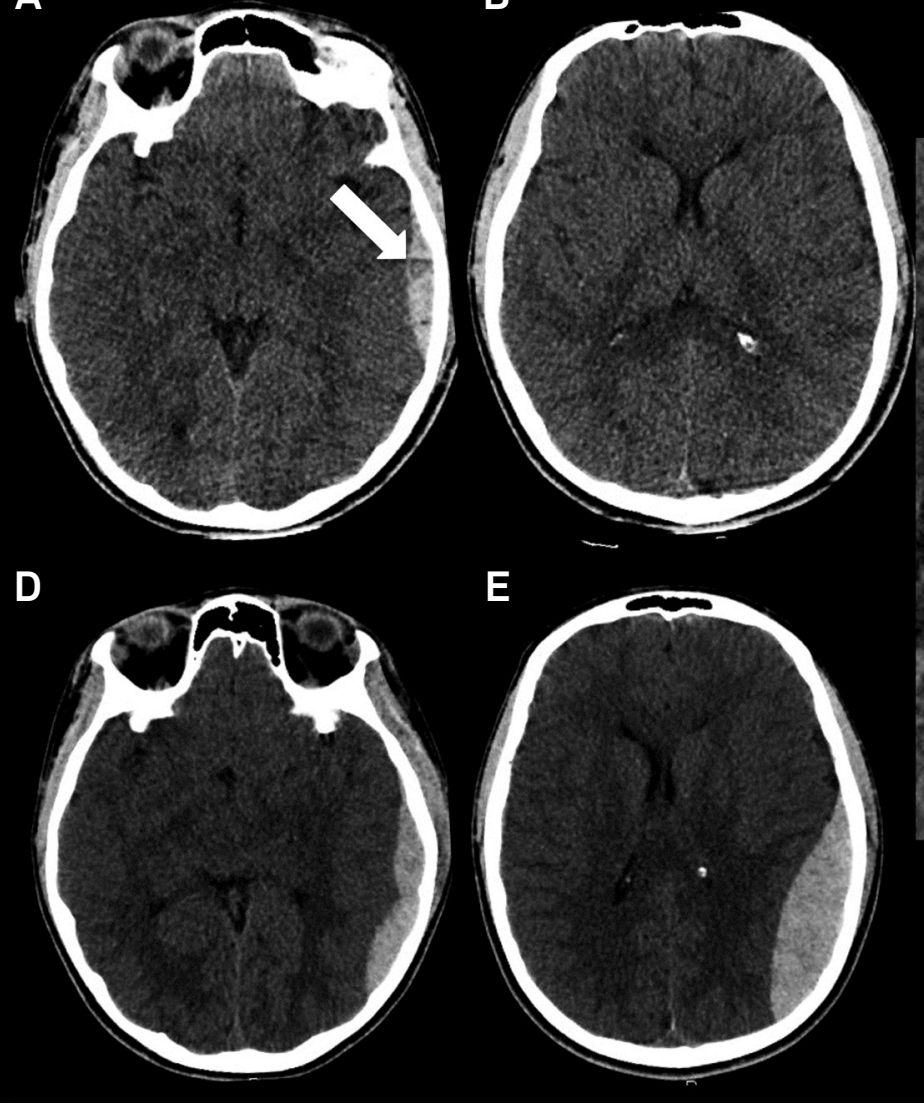

C

$\mathbf{E}$

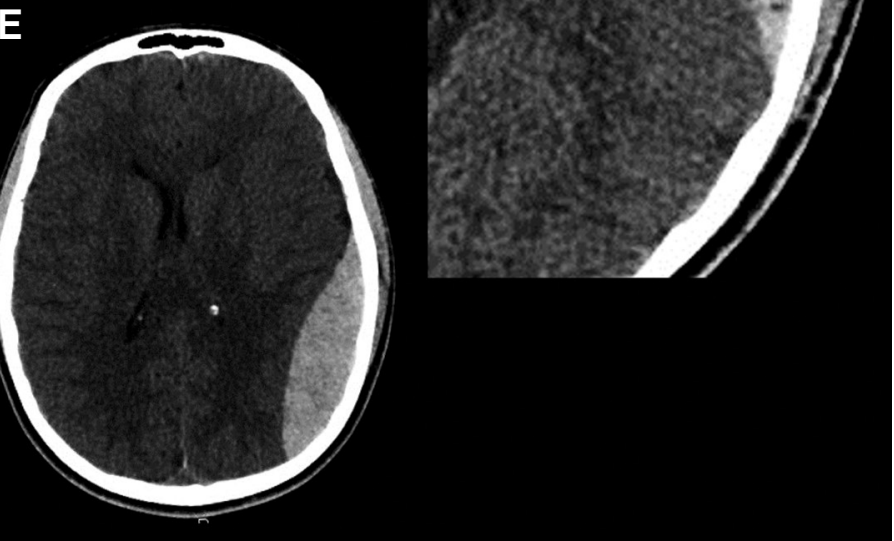

Figure. (A, B and C) Non-contrast CT shows an epidural hematoma (arrow in A) with the swirl signal (arrow in C). After 24 hours, a new non-contrast CT showed an evident hematoma expansion ( $D$ and $E$ ).

\footnotetext{
'Universidade Federal do Espírito Santo, Empresa Brasileira de Serviços Hospitalares, Hospital Universitário Cassiano Antônio de Moraes, Departamento de Neurorradiologia, Vitória ES, Brasil;

${ }^{2}$ Universidade Federal do Espírito Santo, Empresa Brasileira de Serviços Hospitalares, Hospital Universitário Cassiano Antônio de Moraes, Departamento de Radiologia, Vitória ES, Brasil;

${ }^{3}$ Hospital Estadual Jayme Santos Neves, Departamento de Radiologia, Serra ES, Brasil.

Marcos Rosa Júnior (iD https://orcid.org/0000-0001-8668-2804

Correspondence: Marcos Rosa Júnior; Departamento de Neurorradiologia da UFES, Centro de Ciências da Saúde; Avenida Marechal Campos, 1355 - Maruípe; 29043-900 Vitória ES, Brasil. E-mail: marcosrosajr@hotmail.com

Conflict of interest: There is no conflict of interest to declare.

Received 14 September 2018; Received in final form 11 October 2018; Accepted 12 November 2018.
} 


\section{References}

1. Guo C, Liu L, Wang B, Wang Z. Swirl sign in traumatic acute epidural hematoma: prognostic value and surgical management. Neurol Sci. 2017 Dec;38(12):2111-6. https://doi.org/10.1007/s10072-017-3121-4

2. Wagemans BA, Klinkenberg S, Postma AA. Teaching neurolmages: swirl sign and spot sign in intraparenchymal hematoma. Neurology. $2016 \mathrm{Nov;87(18):e225-6.}$ https://doi.org/10.1212/WNL.0000000000003290

3. Rosa Júnior M, Rocha AJ, Saade N, Maia Júnior AC, Gagliardi RJ. Active extravasation of contrast within the hemorrhage (spot sign): a multidetector computed tomography finding that predicts growth and a worse prognosis in non-traumatic intracerebral hemorrhage.
Arq Neuropsiquiatr. 2013 Oct;71(10):791-7. https://doi.org/10.1590/0004-282X20130124

4. Rosa Júnior M, Rocha AJ, Maia Júnior AC, Saade N, Gagliardi RJ. The active extravasation of contrast (spot sign) depicted on multidetector computed tomography angiography might predict structural vascular etiology and mortality in secondary intracranial hemorrhage. J Comput Assist Tomogr. 2015 Mar-Apr;39(2):217-21. https://doi.org/10.1097/RCT.0000000000000182

5. Rosa Júnior M, Rocha AJ, Maia Júnior AC, Saade N, Veiga JC, Romero JM. Contusion contrast extravasation depicted on multidetector computed tomography angiography predicts growth and mortality in traumatic brain contusion. J Neurotrauma. 2016 Jun;33(11):1015-22. https://doi.org/10.1089/neu.2015.4062 